\title{
EFFECTS OF DIFFRACTION AND RADIATION ON A SUBMERGED SPHERE
}

\author{
MATIUR RAHMAN
}

(Received 20 July 2001)

\begin{abstract}
This paper deals with an investigation of the effects of diffraction and radiation on a submerged sphere in water of finite depth $d$. We assume that the fluid is homogeneous, inviscid, and incompressible, and the fluid motion is irrotational. In real situations, the submerged sphere will experience six degrees of freedom (i.e., motions); three translational and three rotational. In this paper, however, we consider a very idealized situation because of the complex nature of the physical problem. Two important motions, namely, the surge (horizontal oscillations) and the heave (vertical oscillations) motions are studied. Our attention is mainly focused on the hydrodynamic coefficients of these motions. The crux of the problem lies entirely on the determination of these coefficients which are inherently related to the determination of the motions of the submerged sphere in regular waves. This type of problem is usually solved by using potential theory, and mathematically, we look for the solution of a velocity potential which satisfies Laplace's equation along with the free surface, body surface, and bottom boundary conditions in conjunction with a radiation condition. This boundary value problem, in fact, consists of two separate problems: (a) diffraction problem and (b) radiation problem.
\end{abstract}

2000 Mathematics Subject Classification. 76R10.

1. Introduction. The motions of a spherical body in a regular gravity wave are investigated in this paper. There are plenty of practical instances where this study can be used. A simple and very useful example in the real world is the motion of a submarine of spherical shape in water of finite or infinite depth if the submarine hull is considered a neutrally buoyant sphere.

The study of waves and wave loading on submerged structures has been the subject of active research since the days of Havelock [4], and in practice, he can be considered as the pioneer in this area of research. A British electrical engineer turned applied mathematician, Havelock contributed tremendously in the field of water waves. Havelock's pioneering work investigating the vertical motion of a floating hemisphere in infinite water depth was extended by Hulme [5] to investigate the added mass and radiation damping. Gray [3] studied the scattering problem of a submerged sphere by expanding Green's function and the associated velocity potential in spherical harmonics.

An analysis of the hydrodynamic problem of linear forces acting on a submerged sphere in an infinite water depth was investigated by Wang [9]. By employing a special series solution, Wang solved the governing equation satisfied by the velocity potential. Wu and Taylor $[10,11]$ considered a submerged spheroid and obtained analytical solutions for the linear forces. An analysis of wave induced drift forces acting on 
a submerged sphere in finite depth was presented by Wu et al. [12]. They used the method of multipole expansions as demonstrated by Thorne [8] to determine the linear velocity potential for a finite water depth. In a recent study, Bora [1] and Bora et al. [2] used the multipole expansion method of Thorne to obtain the velocity potential for the problem of a submerged sphere in finite water depth. In that work the mathematical problem was split into two boundary value problems: a diffraction and a radiation problem.

This paper presents a systematic analysis for calculating the velocity potentials arising in the diffraction and radiation problems due to a submerged sphere in finite water depth. We have evaluated two very important hydrodynamic coefficients inherent to the problem. By using the multipole expansion method, the added mass and radiation damping coefficients are obtained. The mathematics is extremely complex due to the presence of sophisticated mathematical functions namely, spherical Bessel functions and associated Legendre functions which play paramount roles in the solution process. The linear complex algebraic equation plays an important role in the solution process, which determines the important unknown constants. Once these constants are determined, the problem is completely solved. We believe that the combined effects of diffraction and radiation by a submerged sphere in finite water depth have not been investigated before, and to the best of our knowledge this has been significantly absent from all the published literature so far. The determination of the motions using these two coefficients by the combined effects of diffraction and radiation adds a novelty of advancement to our knowledge in this important area of research. We have presented our analytical results in a lucid and very systematic way.

2. Mathematical formulation for the diffraction problem. We assume that the fluid is homogeneous, inviscid and incompressible and the fluid motion is irrotational. The waves are also assumed to be of small amplitude. Here we consider the coefficients related to the motion with two degrees of freedom, namely, the two translational motions in the $x$ and $z$ directions, that is, surge and heave motions, respectively. We consider a surface wave of amplitude $A$ incident on a sphere of radius $a$ submerged in water of finite depth $d$. The body is assumed to have motions with three degrees of freedom in the presence of incident waves with angular frequency $\sigma$. The wave is parallel to the $x$-axis at the time of incidence on the sphere and is propagating along the positive direction.

We consider two sets of coordinate systems. One is a right-handed Cartesian coordinate system $(x, y, z)$, in which the $x-y$ plane coincides with the undisturbed free surface and the $z$-axis is taken vertically downwards from the still water level. The other coordinate system is the spherical coordinate system $(r, \theta, \psi)$ with the origin at the geometric center $(0,0, h)$ of the sphere. The relationship between the coordinate systems is given by $z-h=r \cos \theta, x=r \sin \theta \cos \psi$, and $y=r \sin \theta \sin \psi$ such that $R=\sqrt{x^{2}+y^{2}}, r=\sqrt{R^{2}+(z-h)^{2}}, \tan \theta=R /(z-h)$, for $0 \leq \theta \leq \pi$, and $\tan \psi=y / x$, for $-\pi \leq \psi \leq \pi$.

For an incompressible and inviscid fluid, and for small amplitude wave theory with irrotational motion, we can express the fluid motion by introducing a velocity potential 
$\Phi(r, \theta, \psi, t)$. This $\Phi$ can be written as

$$
\Phi(r, \theta, \psi, t)=\operatorname{Re}\left[\phi(r, \theta, \psi) e^{-i \sigma t}\right],
$$

where Re stands for the real part.

The motion is also assumed to be harmonic. Also, from Bernoulli's equation, we get pressure, $P(r, \theta, \psi, t)$ as

$$
P=-\rho \frac{\partial \Phi}{\partial t}
$$

Now, the problem can be considered as a combination of two fundamental problems: the diffraction problem of an incident wave interacting with a fixed body; and the radiation problem of a body forced to oscillate in otherwise still water. Because of the linearity of the situation, the time-independent velocity potential $\phi(r, \theta, \psi)$ can be decomposed into four velocity potentials $\phi_{I}, \phi_{D}, \phi_{1}$, and $\phi_{3}$ where $\phi_{I}$ is the incident potential, $\phi_{D}$ is the velocity potential due to the diffraction of an incident wave acting on the sphere; and $\phi_{1}$ and $\phi_{3}$ are velocity potentials due to the radiation of surge and heave, respectively.

Thus, $\phi$ can be written as $\phi=\phi_{I}+\phi_{D}+X_{1} \phi_{1}+X_{3} \phi_{3}$, where $X_{1}$ and $X_{3}$ are the displacements for surge and heave motions, respectively. Here $\phi_{I}, \phi_{D}, \phi_{j}, j=1,3$, are all functions of $r, \theta$, and $\psi$ and $X_{j}, j=1,3$, is the independent parameter.

To obtain the velocity potential $\phi$, the following boundary problem is to be solved. Laplace's equation in spherical coordinates:

$$
\nabla^{2} \phi=0
$$

Free surface boundary condition:

$$
\frac{\partial \phi}{\partial z}+K \phi=0 \quad \text { on } z=0 .
$$

Bottom boundary condition:

$$
\frac{\partial \phi}{\partial z}=0, \quad z=d
$$

Radiation condition:

$$
\lim _{R \rightarrow \infty} \sqrt{R}\left(\frac{\partial}{\partial R}-i k_{0}\right) \phi=0
$$

where $K=\sigma^{2} / g$ and $k_{0}$ is the finite depth wavenumber defined by

$$
k_{0} \sinh k_{0} d-K \cosh k_{0} d=0
$$

and the incident and diffraction potentials satisfy the body surface condition

$$
\frac{\partial \phi_{I}}{\partial \mathbf{n}}=-\frac{\partial \phi_{D}}{\partial \mathbf{n}} \quad \text { on } r=a
$$

where $\mathbf{n}$ denotes the normal vector from body surface to fluid. 
The radiation potentials satisfy the body surface condition

(a) for surge motion:

$$
\frac{\partial \phi}{\partial r}=i \sigma \sin \theta \cos \psi \quad \text { on } r=a
$$

(b) for heave motion:

$$
\frac{\partial \phi}{\partial r}=i \sigma \cos \theta \quad \text { on } r=a .
$$

The boundary conditions (2.9) and (2.10) have arisen from the equation

$$
\frac{\partial \phi_{j}}{\partial \mathbf{n}}=(-i \sigma) n_{j}, \quad j=1,3
$$

2.1. Incident potential. The incoming waves of amplitude $A$ and frequency $\sigma$ propagating in the positive $x$-direction can be described by the following incident velocity potential:

$$
\phi_{I}=\frac{A g}{\sigma} \frac{\cosh k_{0}(z-d)}{\cosh k_{0} d} e^{i k_{0} R \cos \psi} .
$$

Using McLachlan [6] and Thorne [8], the incident potential can be expressed in terms of the associated Legendre functions as

$$
\begin{aligned}
\phi_{I}= & \frac{A g}{2 \sigma \cosh k d} \sum_{m=0}^{\infty} \epsilon_{m} i^{m} \cos m \psi \\
& \times\left[e^{k_{0}(h-d)} \sum_{s=m}^{\infty}\left(k_{0} r\right)^{s} \frac{P_{s}^{m}(\cos \theta)}{(s+m) !}+e^{-k_{0}(h-d)} \sum_{s=m}^{\infty}(-1)^{m+s}\left(k_{0} r\right)^{s} \frac{P_{s}^{m}(\cos \theta)}{(s+m) !}\right] \\
= & \frac{A g}{2 \sigma \cosh k_{0} d} \sum_{m=0}^{\infty} \epsilon_{m} i^{m} \cos m \psi \\
& \times \sum_{s=m}^{\infty}\left\{(-1)^{s+m} e^{k_{0}(d-h)}+e^{k_{0}(h-d)}\right\} \frac{\left(k_{0} r\right)^{s}}{(s+m) !} P_{s}^{m}(\cos \theta),
\end{aligned}
$$

where $\epsilon_{0}=1$ and $\epsilon_{m}=2$ for $m \geq 1$, or we can write for our convenience,

$$
\phi_{I}(r, \theta, \psi)=\sum_{m=0}^{\infty} \hat{\phi}_{I}(r, \theta) \cos m \psi,
$$

where

$$
\hat{\phi}_{I}=\frac{A g}{2 \sigma \cosh k_{0} d} \epsilon_{m} i^{m} \sum_{s=m}^{\infty}\left\{(-1)^{s+m} e^{k_{0}(d-h)}+e^{k_{0}(h-d)}\right\} \frac{\left(k_{0} r\right)^{s}}{(s+m) !} P_{s}^{m}(\cos \theta) .
$$

Changing $s$ to $s+m$ and modifying, we have

$$
\hat{\phi}_{I}(r, \theta)=\frac{A g}{\sigma} \epsilon_{m} i^{m} \sum_{s=0}^{\infty} \chi_{s} \frac{\left(k_{0} r\right)^{s+m}}{(s+2 m) !} P_{s+m}^{m}(\cos \theta),
$$


where

$$
\chi_{s}=\frac{(-1)^{s} e^{k_{0}(d-h)}+e^{-k_{0}(d-h)}}{2 \cosh k_{0} d}= \begin{cases}\frac{\cosh k_{0}(d-h)}{\cosh k_{0} d}, & s=0,2,4,6, \ldots, \\ -\frac{\sinh k_{0}(d-h)}{\cosh k_{0} d}, & s=1,3,5, \ldots\end{cases}
$$

Hence, the incident potential $\phi_{I}$ can be written in the final form as

$$
\phi_{I}(r, \theta, \psi)=\sum_{m=0}^{\infty} \frac{A g}{\sigma} \epsilon_{m} i^{m} \sum_{s=0}^{\infty} \chi_{s} \frac{\left(k_{0} r\right)^{s+m}}{(s+2 m) !} P_{s+m}^{m}(\cos \theta) \cos m \psi .
$$

2.2. Diffraction potential. The diffraction velocity potential $\phi_{D}$ satisfies (2.3), (2.4), (2.5), (2.6), and (2.8). We can express this potential by making it $\psi$-independent as follows:

$$
\phi_{D}(r, \theta, \psi)=\sum_{m=0}^{\infty} \hat{\phi}_{D}(r, \theta) \cos m \psi,
$$

where the $\psi$-independent potential is

$$
\hat{\phi}_{D}(r, \theta)=\sum_{n=m}^{\infty} a^{n+2} A_{m n} G_{n}^{m} .
$$

Here $A_{m n}$ are the unknown complex coefficients and $G_{n}^{m}$ are the multipole potentials. Multipole potentials are solutions of Laplace's equation which satisfy the free surface and bottom boundary conditions and behave like outgoing waves from the singular point which in this case is the centre of the sphere.

The potential $G_{n}^{m}$ can be expressed as

$$
\begin{aligned}
G_{n}^{m}= & \frac{P_{n}^{m}(\cos \theta)}{r^{n+1}}+\frac{P_{n}^{m}(\cos \alpha)}{r_{1}^{n+1}}+\frac{1}{(n-m) !} \\
& \times \int_{0}^{\infty} \frac{(K+k)\left[e^{-k(d+H)}+(-1)^{n+m} e^{-k h}\right]}{k \sinh k d-K \cosh k d} k^{n} \cosh k(z-d) J_{m}(k R) d k .
\end{aligned}
$$

The quantities $\alpha$ and $r_{1}$ are defined as

$$
r_{1}=\sqrt{R^{2}+(d+H-z)^{2}}, \quad \tan \alpha=\frac{R}{d+H-z} .
$$

The line integration in the expression for $G_{n}^{m}$ passes under the singular point of the integrand at $k=k_{0}$. The potentials $G_{n}^{m}$ and $\phi_{D}$ satisfy Laplace's equation, free surface condition, bottom surface condition, and the radiation condition.

The second and third terms in (2.21) can be expanded in the region near the body surface into a series of the associated Legendre functions by

$$
\begin{aligned}
& \frac{P_{n}^{m}(\cos \alpha)}{r_{1}^{n+1}}=\sum_{s=0}^{\infty} B_{n s}^{m}\left(\frac{r}{2 H}\right)^{s+m} P_{s+m}^{m}(\cos \theta), \\
& \frac{1}{(n-m) !} \int_{0}^{\infty} \frac{(K+k)\left[e^{-k(d+H)}+(-1)^{n+m} e^{-k h}\right]}{k \sinh k d-K \cosh k d} k^{n} \cosh k(z-d) J_{m}(k R) d k \\
&=\sum_{s=0}^{\infty} C_{s}(n, m)\left(\frac{r}{2 H}\right)^{s+m} P_{s+m}^{m}(\cos \theta),
\end{aligned}
$$


where $B_{n s}^{m}$ and $C_{s}(n, m)$ are given by

$$
\begin{aligned}
B_{n s}^{m} & =\frac{1}{(2 H)^{n+1}} \frac{(s+n+m) !}{(s+2 m) !(n-m) !} \\
C_{s}(n, m) & =\frac{(2 H)^{s+m}}{(n-m) !(s+2 m) !} \int_{0}^{\infty} \frac{(K+k)\left[e^{-k(d+H)}+(-1)^{n+m} e^{-k h}\right]}{k \sinh k d-K \cosh k d} u_{s}(k H) d k,
\end{aligned}
$$

with $u_{s}(k H)$ as

$$
u_{s}(k H)= \begin{cases}\cosh k H, & s=0,2,4, \ldots \\ -\sinh k H, & s=1,3,5, \ldots\end{cases}
$$

Hence, the multipole potentials $G_{n}^{m}$ can finally be written as

$$
G_{n}^{m}=\frac{P_{n}^{m}(\cos \theta)}{r^{n+1}}+\sum_{s=0}^{\infty}\left[B_{n s}^{m}+C_{s}(n, m)\right]\left(\frac{r}{2 H}\right)^{s+m} P_{s+m}^{m}(\cos \theta) .
$$

Using the body surface boundary condition (2.8), we may write

$$
\left.\sum_{n=m}^{\infty} a^{n+2} A_{m n} \frac{\partial G_{n}^{m}}{\partial r}\right|_{r=a}=-\left.\frac{\partial \hat{\phi}_{I}}{\partial r}\right|_{r=a} .
$$

From the expressions for $G_{n}^{m}$ and $\hat{\phi}_{I}$ from (2.26) and (2.16), respectively, we can evaluate $\left.\left(\partial G_{n}^{m} / \partial r\right)\right|_{r=a}$ and $\left.\left(\partial \hat{\phi}_{I} / \partial r\right)\right|_{r=a}$, and using these two expressions in (2.27), we get

$$
\begin{gathered}
\sum_{n=m}^{\infty} A_{m n}\left[-(n+1) P_{n}^{m}(\cos \theta)+\sum_{s=0}^{\infty}\left\{B_{n s}^{m}+C_{s}(n, m)\right\}\left(\frac{a}{2 H}\right)^{s+m}(s+m) a^{n+1} P_{s+m}^{m}(\cos \theta)\right] \\
=-\frac{A g}{\sigma} \epsilon_{m} i^{m} \sum_{s=0}^{\infty} \chi_{s} \frac{\left(k_{0} a\right)^{s+m}}{(s+2 m) !}(s+m) a^{-1} P_{s+m}^{m}(\cos \theta) .
\end{gathered}
$$

Using the orthogonality property of the associated Legendre functions and modifying the result, we arrive at

$$
\sum_{n=m}^{\infty} A_{m n} E_{n s}^{m}=T_{s}^{m} \quad \text { for } s=m, m+1, m+2, \ldots,
$$

where

$$
\begin{aligned}
T_{s}^{m} & =-\frac{A g k_{0}}{\sigma} \epsilon_{m} i^{m}\left(k_{0} a\right)^{s-1} \frac{s}{(s+m) !} \chi_{s-m}, \\
E_{n s}^{m} & =-(n+1) \delta_{n s}+D_{n}^{m}(s-m), \\
D_{n}^{m}(s) & =a^{n+1}(s+m)\left(\frac{a}{2 H}\right)^{s+m}\left[C_{s}(n, m)+B_{n s}^{m}\right] .
\end{aligned}
$$

The diffraction potential $\phi_{D}$ has the final form

$$
\phi_{D}=\sum_{m=0}^{\infty} \sum_{n=m}^{\infty} a^{n+2} A_{m n}\left[\frac{P_{n}^{m}(\cos \theta)}{r^{n+1}}+\sum_{s=0}^{\infty}\left\{B_{n s}^{m}+C_{s}(n, m)\right\}\left(\frac{r}{2 H}\right)^{s+m} P_{s+m}^{m}(\cos \theta)\right] \cos m \psi .
$$


3. Exciting forces. The forces associated with the incident and diffraction potentials are the exciting forces which play a very important role in the wave field for a structure in water. The exciting forces $F_{j}^{(e)}$ can be obtained from

$$
F_{j}^{(e)}=\left.2 i \rho a^{2} \sigma A \int_{o}^{\pi} \int_{0}^{\pi} \phi_{I D}\right|_{r=a} n_{j} \sin \theta d \theta d \psi,
$$

where $j=0$ corresponds to heave exciting force and $j=1$ corresponds to surge exciting force and we have written $\phi_{I D}=\phi_{I}+\phi_{D}$,

$$
n_{j}=-P_{1}^{j}(\cos \theta) \cos j \psi, \quad j=0,1 .
$$

From (2.18) and (2.31), we have

$$
\begin{gathered}
\left.\frac{\partial \phi_{I}}{\partial r}\right|_{r=a}=\sum_{m=0}^{\infty} \frac{A g}{\sigma} \epsilon_{m} i^{m} \sum_{s=0}^{\infty} x_{s}(s+m) \frac{\left(k_{0} a\right)^{s+m}}{a(s+2 m) !} P_{s+m}^{m}(\cos \theta) \cos m \psi, \\
\left.\frac{\partial \phi_{D}}{\partial r}\right|_{r=a}=\sum_{m=0}^{\infty} \sum_{n=m}^{\infty} A_{m n}\left[-(n+1) P_{n}^{m}(\cos \theta)+\sum_{s=0}^{\infty}\left(B_{n s}^{m}+C_{s}\right)\left(\frac{a}{2 H}\right)^{s+m}\right. \\
\left.\times(s+m) a^{n+1} P_{s+m}^{m}(\cos \theta)\right] \cos m \psi .
\end{gathered}
$$

Applying the body surface condition $\partial \phi_{D} / \partial r=-\partial \phi_{I} / \partial r$ at $r=a$ and some simplifications gives

$$
\left.\phi_{I D}\right|_{r=a}=a \sum_{m=0}^{\infty} \sum_{n=m}^{\infty} \frac{2 n+1}{n} A_{m n} P_{n}^{m}(\cos \theta) \cos m \psi .
$$

Now the exciting forces are given by

$$
\begin{aligned}
F_{j}^{(e)} & =-\left.2 i \rho \sigma a^{2} A \int_{0}^{\pi} \int_{0}^{\pi} \phi_{I D}\right|_{r=a} P_{1}^{j}(\cos \theta) \cos j \psi \cos \psi \sin \theta d \theta d \psi \\
& =-\frac{2 i \rho \sigma a^{2} A \pi}{\epsilon_{j}} \int_{0}^{\pi} \sum_{n=j}^{\infty} a \frac{2 n+1}{n} A_{j n} P_{n}^{j}(\cos \theta) \sin \theta d \theta,
\end{aligned}
$$

where $\epsilon_{j}=1$ for $j=0, \epsilon_{j}=2$ for $j \geq 1$.

Using the orthogonality property of the associated Legendre functions,

$$
F_{j}^{(e)}=-2 i \rho \sigma \pi a^{3} A \epsilon_{j} 2 \frac{(1+j) !}{(1-j) !} A_{j 1}=-4 i \rho \sigma \pi a^{3} A A_{j 1} .
$$

Hence, the horizontal force, that is, the surge exciting force $F_{x}^{(e)}=f_{x d}$ is given by

$$
f_{x d}=-4 i \rho \sigma \pi A a^{3} A_{11} .
$$

The vertical force, that is, the heave exciting force $F_{z}^{(e)}=f_{z d}$ is given by

$$
f_{z d}=-4 i \rho \sigma \pi A a^{3} A_{01} .
$$

Non-dimensionalizing the forces given by (3.7) and (3.8), we can write the nondimensional forces as

$$
\frac{f_{x d}}{4 i \rho \sigma A \pi a^{3}}=-A_{11}, \quad \frac{f_{z d}}{4 i \rho \sigma A \pi a^{3}}=-A_{01} .
$$


4. Radiation problem. Having solved the diffraction problem for the submerged sphere, we now turn our attention to the radiation problem. As mentioned earlier we will consider surge and heave potentials only. All these potentials satisfy the same set of equations except for the body boundary condition which is different for each motion. Both being related with translational motions, surge and heave potentials have resemblance in their expressions. Hence we proceed to find the expression for surge and heave potentials at the same time and then evaluate them using the respective boundary conditions. One very important point to note is that due to the body symmetry of a sphere, no moment acts upon the body.

The radiation velocity potential $\phi_{m}$ must satisfy

$$
\begin{aligned}
& \nabla^{2} \phi_{m}=0 \quad \text { in the fluid, } \\
& \frac{\partial \phi_{m}}{\partial z}+K \phi_{m}=0 \quad \text { on } z=0, \\
& \frac{\partial \phi_{m}}{\partial z}=0 \quad \text { on } z=d, \\
& \frac{\partial \phi_{m}}{\partial r}=(-i \sigma) n_{j}, \quad j=1,3 \text { on } r=a, \\
& \lim _{R \rightarrow \infty} R^{1 / 2}\left\{\frac{\partial}{\partial R}-i k\right\} \phi_{m}=0 .
\end{aligned}
$$

The kinematic boundary condition on the body surface for the radiation problem in the case of surge and heave motions can be written as

$$
\frac{\partial \phi_{m}}{\partial r}=i \sigma P_{1}^{m}(\cos \theta) \cos m \psi
$$

where $m=0$ corresponds to heave motion and $m=1$ to surge motion.

The $\psi$-dependence of $\phi_{m}$ can be removed by assuming

$$
\phi_{m}(r, \theta, \psi)=\hat{\phi}_{m}(r, \theta) \cos m \psi .
$$

The velocity potential $\hat{\phi}_{m}(r, \theta)$ will be expanded in multipole potentials which have already been discussed while dealing with the diffraction potential. Now, from Thorne [8, Section 5], removing the time dependence term,

$$
\begin{aligned}
\hat{\phi}_{m}(r, \theta)= & \frac{P_{n}^{m}(\cos \theta)}{r^{n+1}}+\frac{(-1)^{n+m-1}}{(n-m) !} \int_{0}^{\infty} \frac{K+k}{K-k} k^{n} e^{-k(z+d)} J_{m}(k R) d k \\
& +i \frac{(-1)^{m+n}}{(n-m) !} 2 \pi K^{n+1} e^{-k(z+d)} J_{m}(K R),
\end{aligned}
$$

$\hat{\phi}_{m}$ can be finally expressed as

$$
\begin{aligned}
\hat{\phi}_{m}(r, \theta)= & \frac{P_{n}^{m}(\cos \theta)}{r^{n+1}}+\sum_{s=m}^{\infty} \frac{(-1)^{m+s-1}}{(n-m) !(s+m) !} r^{s} P_{s}^{m}(\cos \theta) P V \int_{0}^{\infty} \frac{K+k}{K-k} k^{n+s} e^{-2 k d} d k \\
& +i \sum_{s=m}^{\infty} \frac{(-1)^{n+s}}{(n-m) !(s+m) !} 2 \pi K^{n+s+1} e^{-2 K d} r^{s} P_{s}^{m}(\cos \theta),
\end{aligned}
$$


where $P V$ means the principal value of the integral is to be considered. Alternately, we can write $\hat{\phi}_{m}$ as

$$
\hat{\phi}_{m}(r, \theta)=\frac{P_{n}^{m}(\cos \theta)}{r^{n+1}}+\sum_{s=m}^{\infty}\left[A_{s}+i B_{s}\right] r^{s} P_{n}^{m}(\cos \theta),
$$

where

$$
\begin{aligned}
& A_{s}=\frac{(-1)^{m+s-1}}{(n-m) !(s+m) !} \int_{0}^{\infty} \frac{K+k}{K-k} k^{n+s} e^{-2 k d} d k, \\
& B_{s}=\frac{(-1)^{n+s}}{(n-m) !(s+m) !} 2 \pi K^{n+s+1} e^{-2 K d} .
\end{aligned}
$$

Hence the radiation potential $\phi_{m}$ can be written as

$$
\phi_{m}(r, \theta, \psi)=\left[\frac{P_{n}^{m}(\cos \theta)}{r^{n+1}}+\sum_{s=m}^{\infty}\left(A_{s}+i B_{s}\right) r^{s} P_{s}^{m}(\cos \theta)\right] \cos m \psi .
$$

Applying the body boundary condition for $m=0,1$,

$$
i \sigma P_{1}^{m}(\cos \theta)=\left[\frac{-(n+1) P_{n}^{m}(\cos \theta)}{a^{n+2}}+\sum_{s=m}^{\infty}\left(A_{s}+i B_{s}\right) s a^{s-1} P_{s}^{m}(\cos \theta)\right] .
$$

After simplifying, and using the orthogonality of the associated Legendre functions, and making some re-arrangements,

$$
\begin{aligned}
& \sum_{n=m}^{\infty} \frac{2 n a^{n-1}}{2 n+1} \frac{(n+m) !}{(n-m) !} A_{n}=\frac{2(n+1)}{(2 n+1) a^{n+2}} \frac{(n+m) !}{(n-m) !} \\
& \sum_{n=m}^{\infty} \frac{2 n a^{n-1}}{2 n+1} \frac{(n+m) !}{(n-m) !} B_{n}=\frac{2}{3} \sigma \frac{(1+m) !}{(1-m) !}
\end{aligned}
$$

5. Determination of hydrodynamic coefficients and motion. The coefficients related with the radiation play a big role in allowing us to know the impact of motions due to radiation. The evaluation of added-mass and damping coefficients is of utmost importance in analyzing the contribution of radiation to the total boundary value problem.

5.1. Surge hydrodynamic coefficients. From Sarpkaya and Isaacson [7], the components of the radiated force can be written as

$$
F_{i}^{(R)}=-\sum_{j}\left(\mu_{i j} \frac{\partial^{2} X_{j}}{\partial t^{2}}+\lambda_{i j} \frac{\partial X_{j}}{\partial t}\right),
$$

where $\mu_{i j}$ and $\lambda_{i j}$ are, respectively, called the added-mass and damping coefficients. Those coefficients are taken to be real and are termed added-mass and damping coefficients, respectively, since they assume corresponding roles in the equations of motion. 
The equation of motion can be written as (Newton's law of motion)

$$
\left(M_{i j}+\mu_{i j}\right) \frac{\partial^{2} X_{j}}{\partial t^{2}}+\lambda_{i j} \frac{\partial X_{j}}{\partial t}+C_{i j} X_{j}=F_{i}^{(e)},
$$

where $M_{i j}$ is the mass matrix, $C_{i j}$ the hydrodynamic stiffness matrix and $F_{i}^{(e)}$ are the exciting forces associated with the diffraction potential.

The exciting force can be considered as the forcing function of the motion. It is emphasized that this equation relates to an unrestricted floating or submerged body. The added-mass coefficients $\mu_{i j}$ are analogous to those for a body accelerating in an unbounded fluid, but they are not the same. The damping coefficients $\lambda_{i j}$ are associated with a net outward flux of energy in the radiated waves and thus represent only damping due to (radiating) fluid motion. The coefficients $\mu_{i j}$ and $\lambda_{i j}$ are not dimensionless but possess appropriate dimensions.

The radiated force $F_{r 1}$ due to the surge motion can be written as the real part of $f_{r 1} e^{-i \sigma t}$ where $f_{r 1}$ is given by

$$
\begin{aligned}
f_{r 1} & =2 i \rho a^{2} \sigma A \int_{0}^{\pi} \int_{0}^{\pi} \hat{X}_{1} \phi_{1}(a, \theta, \psi) n_{1} \sin \theta d \theta d \psi \\
& =-2 i \rho a^{2} \sigma A \int_{0}^{\pi} \int_{0}^{\pi} \hat{X}_{1} \phi_{1}(a, \theta, \psi) \sin ^{2} \theta \cos \psi d \theta d \psi .
\end{aligned}
$$

This radiated force can be conveniently decomposed into components in phase with the velocity and the acceleration,

$$
F_{r 1}=-\left(\mu_{11} \frac{\partial^{2} X_{1}}{\partial t^{2}}+\lambda_{11} \frac{\partial X_{1}}{\partial t}\right)
$$

where $X_{1}=\operatorname{Re}\left\{\hat{X}_{1} e^{-i \sigma t}\right\}$. Hence, we can write

$$
f_{r 1}=-\left\{\left(-\sigma^{2}\right) \mu_{11} \hat{X}_{1}+\lambda_{11}(-i \sigma) \hat{X}_{1}\right\}=\left\{\sigma^{2} \mu_{11} \hat{X}_{1}+i \sigma \lambda_{11} \hat{X}_{1}\right\} .
$$

Then equating relations (5.3) and (5.5), we get

$$
\sigma^{2} \mu_{11} \hat{X}_{1}+i \sigma \lambda_{11} \hat{X}_{1}=-2 i \rho a^{2} \sigma A \int_{0}^{\pi} \int_{0}^{\pi} \hat{X}_{1} \phi_{1}(a, \theta, \psi) \sin ^{2} \theta \cos \psi d \theta d \psi
$$

which will yield the following after cancelling $\hat{X}_{1}$ throughout

$$
\mu_{11}+i \frac{\lambda_{11}}{\sigma}=-\frac{2 i \rho a^{2} A}{\sigma} \int_{0}^{\pi} \int_{0}^{\pi} \phi_{1}(a, \theta, \psi) \sin ^{2} \theta \cos \psi d \theta d \psi
$$

Hence, equating the real and imaginary parts, the added-mass and damping coefficients are, respectively, given by

$$
\begin{aligned}
& \mu_{11}=-\frac{2 \rho A a^{2}}{\sigma} \int_{0}^{\pi} \int_{0}^{\pi} \operatorname{Re}\left[i \phi_{1}(a, \theta, \psi)\right] \sin ^{2} \theta \cos \psi d \theta d \psi, \\
& \lambda_{11}=-2 \rho A a^{2} \int_{0}^{\pi} \int_{0}^{\pi} \operatorname{Im}\left[i \phi_{1}(a, \theta, \psi)\right] \sin ^{2} \theta \cos \psi d \theta d \psi .
\end{aligned}
$$


The surge potential $\phi_{1}(r, \theta, \psi)$ at $r=a$ can be written from (4.8) as

$$
\phi_{1}(a, \theta, \psi)=\left[\frac{P_{n}^{1}(\cos \theta)}{a^{n+1}} \sum_{n=1}^{\infty}\left(A_{n}+B_{n}\right) a^{n} P_{n}^{1}(\cos \theta)\right] \cos \psi .
$$

Hence using (5.9) in (5.7) and (5.8) and simplifying by the use of the associated Legendre functions, we obtain the added-mass and damping coefficients as

$$
\begin{aligned}
\mu_{11} & =-\frac{2 \rho A a^{2}}{\sigma} \int_{0}^{\pi} \int_{0}^{\pi}\left[\sum_{n=1}^{\infty}-B_{n} a^{n} P_{n}^{1}(\cos \theta)\right] \cos \psi \times P_{1}^{1}(\cos \theta) \sin \theta \cos \psi d \theta d \psi \\
& =\frac{4}{3} \frac{\rho a^{3} \pi A}{\sigma} B_{1}, \\
\lambda_{11} & =-2 \rho A a^{2} \int_{0}^{\pi} \int_{0}^{\pi}\left[\frac{P_{n}^{1}(\cos \theta)}{a^{n+1}}+\sum_{n=1}^{\infty} A_{n} a^{n} P_{n}^{1}(\cos \theta)\right] \cos \psi \times P_{1}^{1}(\cos \theta) \sin \theta d \theta d \psi \\
& =-\frac{4}{3} \rho \pi A\left[1+A_{1} a^{3}\right] .
\end{aligned}
$$

Or else we can represent $\mu_{11}$ and $\lambda_{11}$ as

$$
\frac{\mu_{11}}{(3 / 4)\left(\rho a^{3} \pi A / \sigma\right)}=B_{1}, \quad \frac{\lambda_{11}}{(4 / 3) \rho \pi A}=-\left[1+A_{1} a^{3}\right] .
$$

Using Newton's law of motions, that is, mass times acceleration $=$ the external forces, we get

$$
M_{11} \frac{\partial^{2} X_{1}}{\partial t^{2}}=-\mu_{11} \frac{\partial^{2} X_{1}}{\partial t^{2}}-\lambda_{11} \frac{\partial X_{1}}{\partial t}+F_{x}^{(e)}
$$

where $M_{11}$ is the mass of the displaced fluid, the first two terms on the right-hand side are due to the radiated force in the $x$-direction in which $\mu_{11}$ is the surge added mass, $\lambda_{11}$ the surge damping coefficient and $F_{x}^{(e)}$ the $x$-component of the exciting force. Although elementary in form, (5.13) plays a fundamental role in the oscillating system. Therefore we must always cite the form of the equation.

In complex form, the equation of motion can be summed up as

$$
\left(M_{11}+\mu_{11}\right)(-i \sigma)^{2} \hat{X}_{1}+(-i \sigma) \hat{X}_{1} \lambda_{11}=f_{x d}
$$

which simplifies to

$$
\left(M_{11}+\mu_{11}\right) \sigma^{2} \hat{X}_{1}+i \sigma \hat{X}_{1} \lambda_{11}=-f_{x d}
$$

where

$$
f_{x d}=-2 i \rho \sigma a^{2} A \int_{0}^{\pi} \int_{0}^{\pi} \phi_{I D}(a, \theta, \psi) \sin ^{2} \theta \cos \psi d \theta d \psi .
$$

That gives us

$$
\hat{X}_{1}=-\frac{f_{x d}}{\sigma^{2}\left(M_{11}+\mu_{11}+i \lambda_{11} / \sigma\right)} .
$$

The amplitude of the surge oscillation is thus determined by (5.17). This solution to this expression is inherently connected to the added mass and radiation damping coefficients. 
5.2. Heave hydrodynamic coefficients. The radiated force $F_{r 3}$ due to the heave motion can be written as the real part of $f_{r 3} e^{-i \sigma t}$ where $f_{r 3}$ is given by

$$
f_{r 3}=-2 i \rho a^{2} A \sigma \int_{0}^{\pi} \int_{0}^{\pi} \hat{X}_{3} \phi_{3}(a, \theta, \psi) \sin \theta \cos \theta d \theta d \psi .
$$

Considering $X_{3}=\operatorname{Re}\left\{\hat{X}_{3} e^{-i \sigma t}\right\}$, we have, proceeding as in Section 5.1,

$$
\mu_{33}+i \frac{\lambda_{33}}{\sigma}=-\frac{2 i \rho A a^{2}}{\sigma} \int_{0}^{\pi} \int_{0}^{\pi} \phi_{3}(a, \theta, \psi) \sin \theta \cos \theta d \theta d \psi,
$$

where $\mu_{33}$ and $\lambda_{33}$ are the heave added-mass and damping coefficient due to heave motion, respectively. Hence,

$$
\begin{aligned}
& \mu_{33}=-\frac{2 \rho A a^{2}}{\sigma} \int_{0}^{\pi} \int_{0}^{\pi} \operatorname{Re}\left[i \phi_{3}(a, \theta, \psi)\right] \sin \theta d \theta d \psi, \\
& \lambda_{33}=-2 \rho A a^{2} \int_{0}^{\pi} \int_{0}^{\pi} \operatorname{Im}\left[i \phi_{3}(a, \theta, \psi)\right] \sin \theta \cos \theta d \theta d \psi .
\end{aligned}
$$

The heave potential $\phi_{3}(r, \theta, \psi)$ at $r=a$ can be written from (4.8) as

$$
\phi_{3}(a, \theta, \psi)=\frac{P_{n}^{0}(\cos \theta)}{a^{n+1}}+\sum_{n=0}^{\infty} D_{n} a^{n} P_{n}^{0}(\cos \theta), \quad D_{n}=A_{n}+i B_{n} .
$$

Therefore, using (5.21) in (5.19) and (5.20) and simplifying with the associated Legendre functions, we obtain the heave coefficients as

$$
\begin{aligned}
\mu_{33} & =-\frac{2 \rho a^{2} A}{\sigma} \int_{0}^{\pi} \int_{0}^{\pi}\left(\sum_{n=0}^{\infty} B_{n} a^{n} P_{n}^{0}(\cos \theta)\right) \times P_{1}^{0}(\cos \theta) \sin \theta d \theta d \psi \\
& =\frac{4}{3} \frac{\rho a^{3} \pi A}{\sigma} B_{1} \\
\lambda_{33} & =-\frac{2 \rho a^{2} A}{\sigma} \int_{0}^{\pi} \int_{0}^{\pi}\left[\frac{P_{n}^{0}(\cos \theta)}{a^{n+1}}+\sum_{n=0}^{\infty} A_{n} a^{n} P_{n}^{0}(\cos \theta)\right] P_{1}^{0}(\cos \theta) \sin \theta d \theta d \psi \\
& =-\frac{4}{3} \frac{\rho \pi A}{\sigma}\left(1+A_{1} a^{3}\right) .
\end{aligned}
$$

Or else we can represent $\mu_{33}$ and $\lambda_{33}$ as

$$
\frac{\mu_{33}}{(4 / 3) \rho a^{3} A \pi}=B_{1}, \quad \frac{\lambda_{33}}{(4 / 3)(\rho A \pi / \sigma)}=-\left[1+A_{1} a^{3}\right] .
$$

The equation of motion (Newton's law of motion) in complex form can be written as

$$
v_{33} \hat{X}_{3}+\left(M_{33}+\mu_{33}\right)(-i \sigma)^{2} \hat{X}_{3}+(-i \sigma) \hat{X}_{3} \lambda_{33}=f_{z d},
$$

where $v_{33}$ is the restoring coefficient which is a known quantity, $M_{33}$ is the mass of the displaced fluid and

$$
f_{z d}=-2 i \rho \sigma a^{2} A \int_{0}^{\pi} \int_{0}^{\pi} \phi_{I D}(a, \theta, \psi) \sin \theta \cos \theta d \theta d \psi .
$$


Hence

$$
\hat{X}_{3}=\frac{f_{z d}}{v_{33}-\sigma^{2}\left(M_{33}+\mu_{33}+i\left(\lambda_{33} / \sigma\right)\right)} .
$$

It is to be noted here that the amplitude of the heave oscillation is assumed to have a restoring force component in the vertical direction, and has been given by (5.28). The evaluation of this amplitude is connected with the determination of the added mass coefficient and the radiation damping which makes the problem interesting. Once the surge amplitude and the heave amplitude have been completely determined, the total forces on the submerged sphere due to diffraction and radiation can be determined. The next section deals with this investigation.

6. Evaluation of forces. This section is concerned with the evaluation of wave forces due to the combined effects of diffraction and radiation. To the best of our knowledge, the following analysis has not been reported in the existing literature. Therefore, we present the analysis in this section. We find the forces acting along the $x$ and $z$ directions. The component of the horizontal force $f_{x}$ can be computed from

$$
f_{x}=f_{x d}+f_{x 1}
$$

where $f_{x d}$ is the $x$-component of the diffraction force and $f_{x 1}$ the force due to surge motion. The mathematical expression for each case is given by

$$
\begin{aligned}
& f_{x d}=-2 i \rho \sigma a^{2} A \int_{0}^{\pi} \int_{0}^{\pi} \phi_{I D}(a, \theta, \psi) \sin ^{2} \theta \cos \psi d \theta d \psi, \\
& f_{x 1}=-2 i \rho \sigma a^{2} A \hat{X}_{1} \int_{0}^{\pi} \int_{0}^{\pi} \phi_{1}(a, \theta, \psi) \sin ^{2} \theta \cos \psi d \theta d \psi .
\end{aligned}
$$

It is to be emphasized here that the double integrals look very simple but the integrands are complicated expressions. Considerable efforts have been made in the integration process as can be seen in the following.

The vertical force component $f_{z}$ can be written as

$$
f_{z}=f_{z d}+f_{z 3}
$$

where $f_{z d}$ is the $z$-component of diffraction force and $f_{z 3}$ the force due to the heave motion. The mathematical expression for each case is given by

$$
\begin{aligned}
& f_{z d}=-2 i \rho \sigma a^{2} A \int_{0}^{\pi} \int_{0}^{\pi} \phi_{I D}(a, \theta, \psi) \sin \theta \cos \theta d \theta d \psi, \\
& f_{z 3}=-2 i \rho \sigma a^{2} A \hat{X}_{3} \int_{0}^{\pi} \int_{0}^{\pi} \phi_{3}(a, \theta, \psi) \sin \theta \cos \theta d \theta d \psi .
\end{aligned}
$$

Similar efforts have also been made in the evaluation of these double integrals with complicated expressions as integrands. The final solutions are summarized in the following. 
Substituting the value of $\phi_{1}(a, \theta, \psi)$ from (5.10) into (6.3), we can evaluate $f_{x 1}$ as

$$
\begin{aligned}
f_{x 1}= & -2 i \rho \sigma a^{2} \pi A \hat{X}_{1} \int_{0}^{\pi} \int_{0}^{\pi}\left[\frac{P_{n}^{1}(\cos \theta)}{a^{n+1}}+\sum_{n=1}^{\infty} D_{n} a^{n} P_{n}^{1}(\cos \theta)\right] \cos \psi \\
& \times P_{1}^{1}(\cos \theta) \cos \psi \sin \theta d \theta d \psi \\
= & -\frac{4}{3} i \rho \pi A \sigma \hat{X}_{1}\left(1+D_{1} a^{3}\right) .
\end{aligned}
$$

Substituting the value of $\phi_{3}(a, \theta, \psi)$ from (5.22) into (6.6), we can evaluate $f_{z 3}$ as

$$
\begin{aligned}
f_{z 3} & =-2 i \rho \sigma a^{2} A \hat{X}_{3} \int_{0}^{\pi} \int_{0}^{\pi}\left[\frac{P_{n}^{0}(\cos \theta)}{a^{n+1}}+\sum_{n=0}^{\infty} D_{n} a^{n} P_{n}^{0}(\cos \theta)\right] P_{1}^{0}(\cos \theta) \sin \theta d \theta d \psi \\
& =-\frac{4}{3} A i \rho \sigma \pi \hat{X}_{3}\left[1+D_{1} a^{3}\right] .
\end{aligned}
$$

Hence, the total force along the $x$-axis is

$$
f_{x}=f_{x d}+f_{x 1}=-4 i \rho \sigma \pi a^{3} A_{11}-\frac{4}{3} i \rho \pi \sigma \hat{X}_{1}\left(1+D_{1} a^{3}\right) .
$$

Similarly, the total force along the $z$-axis is

$$
f_{z}=f_{z d}+f_{z 3}=-4 i \rho \sigma \pi a^{3} A A_{01}-\frac{4}{3} i \rho \sigma \pi A \hat{X}_{3}\left(1+D_{1} a^{3}\right),
$$

where $\hat{X}_{1}$ and $\hat{X}_{3}$ are given by (5.17) and (5.28) and in the following we have rewritten them for ready reference only.

$$
\hat{X}_{1}=-\frac{f_{x d}}{\sigma^{2}\left(M_{11}+\mu_{11}+i\left(\lambda_{11} / \sigma\right)\right)}, \quad \hat{X}_{3}=\frac{f_{z d}}{v_{33}-\sigma^{2}\left(M_{33}+\mu_{33}+i\left(\lambda_{33} / \sigma\right)\right)}
$$

with $\mu_{11}, \lambda_{11}, \mu_{33}$, and $\lambda_{33}$ as already obtained. So, (6.9) and (6.10), respectively, give us the total horizontal and vertical forces due to the combined effect of diffraction and radiation. The evaluation of the forces along the $x$ and $z$ axes helps us in understanding the combined effect of diffraction and radiation.

7. Results and discussions. Exciting force coefficients obtained for the submerged sphere give good comparison with the results obtained by Wang [9] for infinite water depth. In long waves $(K a<0.1)$, the shallow water heave exciting force at the fixed submergence $h / a=1.25$ reduces significantly from that in deep water. The converse is true for surge exciting forces where the values in water of depth $2.5 \mathrm{a}$ are more than double of those in depth $20 a$.

Tables 7.1, 7.2, 7.3, and 7.4 present the results for the added-mass and damping coefficients for both surge and heave motions for different submergence values. The results show good agreement with those obtained by Wang [9]. From Tables 7.1 and 7.3, we see that the added-mass $\mu_{11}$ and $\mu_{33}$ steadily decrease after reaching the maximum values in the range $0.4 \leq K a \leq 0.5$. After $K a=1.5$, they vary very insignificantly. 
TABLE 7.1. Surge added-mass $\mu_{11}$ for different submergence $(h / a)$ values.

\begin{tabular}{lcccc}
\hline & $\leftarrow$ & & $h / a$ & $\rightarrow$ \\
$K a$ & 1.5 & 1.75 & 2.0 & 3.0 \\
\hline 0 & 0.5287 & 0.5179 & 0.5118 & 0.5034 \\
0.1 & 0.5403 & 0.5266 & 0.5187 & 0.5066 \\
0.2 & 0.5545 & 0.5363 & 0.5255 & 0.5082 \\
0.3 & 0.5656 & 0.5422 & 0.5283 & 0.5069 \\
0.4 & 0.5693 & 0.5416 & 0.5255 & 0.5030 \\
0.5 & 0.5646 & 0.5347 & 0.5187 & 0.4986 \\
0.6 & 0.5527 & 0.5234 & 0.5092 & 0.4949 \\
0.7 & 0.5359 & 0.5107 & 0.4989 & 0.4920 \\
0.8 & 0.5160 & 0.4966 & 0.4895 & 0.4905 \\
0.9 & 0.4962 & 0.4841 & 0.4815 & 0.4893 \\
1.0 & 0.4776 & 0.4732 & 0.4752 & 0.4896 \\
1.2 & 0.4475 & 0.4578 & 0.4675 & 0.4903 \\
1.4 & 0.4286 & 0.4497 & 0.4648 & 0.4915 \\
1.6 & 0.4189 & 0.4475 & 0.4652 & 0.4925 \\
1.8 & 0.4158 & 0.4481 & 0.4676 & 0.4930 \\
2.0 & 0.4171 & 0.4505 & 0.4698 & 0.4938 \\
3.0 & 0.4381 & 0.4653 & 0.4787 & 0.4950 \\
4.0 & 0.4523 & 0.4721 & 0.4825 & 0.4955 \\
5.0 & 0.4582 & 0.4750 & 0.4839 & 0.4966 \\
\hline
\end{tabular}

TABLE 7.2. Surge damping coefficients $\lambda_{11}$ for different submergence $(h / a)$ values.

\begin{tabular}{|c|c|c|c|c|}
\hline & $\leftarrow$ & & $h / a$ & $\rightarrow$ \\
\hline Ka & 1.5 & 1.75 & 2.0 & 3.0 \\
\hline 0 & 0. & 0. & 0. & 0. \\
\hline 0.1 & 0.0018 & 0.0017 & 0.0016 & 0.0013 \\
\hline 0.2 & 0.0113 & 0.0098 & 0.0088 & 0.0057 \\
\hline 0.3 & 0.0285 & 0.0237 & 0.0200 & 0.0106 \\
\hline 0.4 & 0.0506 & 0.0398 & 0.0317 & 0.0138 \\
\hline 0.5 & 0.0734 & 0.0544 & 0.0412 & 0.0147 \\
\hline 0.6 & 0.0934 & 0.0655 & 0.0472 & 0.0138 \\
\hline 0.7 & 0.1082 & 0.0722 & 0.0496 & 0.0120 \\
\hline 0.8 & 0.1172 & 0.0745 & 0.0489 & 0.0099 \\
\hline 0.9 & 0.1205 & 0.0733 & 0.0460 & 0.0076 \\
\hline 1.0 & 0.1190 & 0.0695 & 0.0418 & 0.0057 \\
\hline 1.2 & 0.1063 & 0.0574 & 0.0317 & 0.0030 \\
\hline 1.4 & 0.0873 & 0.0438 & 0.0223 & 0.0014 \\
\hline 1.6 & 0.0678 & 0.0318 & 0.0148 & 0.0006 \\
\hline 1.8 & 0.0504 & 0.0220 & 0.0094 & 0.0003 \\
\hline 2.0 & 0.0363 & 0.0148 & 0.0058 & 0.0001 \\
\hline 3.0 & 0.0053 & 0.0015 & 0.0004 & 0.0000 \\
\hline 4.0 & 0.0005 & 0.0001 & 0.0000 & 0.0000 \\
\hline 5.0 & 0.0000 & 0.0000 & 0.0000 & 0.0000 \\
\hline
\end{tabular}


TABLE 7.3. Heave added-mass $\mu_{33}$ for different submergence $(h / a)$ values.

\begin{tabular}{ccccc}
\hline & $\leftarrow$ & & $h / a$ & $\rightarrow$ \\
$K a$ & 1.5 & 1.75 & 2.0 & 3.0 \\
\hline 0.0 & 0.5586 & 0.5362 & 0.5239 & 0.5070 \\
0.1 & 0.5834 & 0.5539 & 0.5375 & 0.5131 \\
0.2 & 0.6139 & 0.5742 & 0.5518 & 0.5166 \\
0.3 & 0.6365 & 0.5859 & 0.5570 & 0.5133 \\
0.4 & 0.6421 & 0.5831 & 0.5506 & 0.5055 \\
0.5 & 0.6272 & 0.5667 & 0.5350 & 0.4969 \\
0.6 & 0.5955 & 0.5414 & 0.5147 & 0.4895 \\
0.7 & 0.5541 & 0.5127 & 0.4939 & 0.4845 \\
0.8 & 0.5095 & 0.4846 & 0.4752 & 0.4890 \\
0.9 & 0.4680 & 0.4598 & 0.4598 & 0.4794 \\
1.0 & 0.4316 & 0.4394 & 0.4481 & 0.4793 \\
1.2 & 0.3788 & 0.4123 & 0.4346 & 0.4805 \\
1.4 & 0.3497 & 0.3998 & 0.4306 & 0.4827 \\
1.6 & 0.3381 & 0.3971 & 0.4321 & 0.4847 \\
1.8 & 0.3374 & 0.4000 & 0.4362 & 0.4863 \\
2.0 & 0.3428 & 0.4055 & 0.4412 & 0.4874 \\
3.0 & 0.3852 & 0.4331 & 0.4587 & 0.4901 \\
4.0 & 0.4091 & 0.4457 & 0.4654 & 0.4910 \\
5.0 & 0.4203 & 0.4513 & 0.4686 & 0.4918 \\
\hline
\end{tabular}

TABLE 7.4. Heave damping coefficients $\lambda_{33}$ for different submergence $(h / a)$ values.

\begin{tabular}{|c|c|c|c|c|}
\hline & $\leftarrow$ & & $h / a$ & $\rightarrow$ \\
\hline Ka & 1.5 & 1.75 & 2.0 & 3.0 \\
\hline 0 & 0. & 0. & 0. & 0. \\
\hline 0.1 & 0.0040 & 0.0036 & 0.0033 & 0.0026 \\
\hline 0.2 & 0.0245 & 0.0208 & 0.0182 & 0.0116 \\
\hline 0.3 & 0.0631 & 0.0505 & 0.0416 & 0.0215 \\
\hline 0.4 & 0.1129 & 0.0847 & 0.0658 & 0.0276 \\
\hline 0.5 & 0.1627 & 0.1149 & 0.0848 & 0.0293 \\
\hline 0.6 & 0.2037 & 0.1361 & 0.0958 & 0.0275 \\
\hline 0.7 & 0.2304 & 0.1473 & 0.0991 & 0.0237 \\
\hline 0.8 & 0.2423 & 0.1490 & 0.0964 & 0.0193 \\
\hline 0.9 & 0.2414 & 0.1439 & 0.0896 & 0.0150 \\
\hline 1.0 & 0.2318 & 0.1340 & 0.0805 & 0.0115 \\
\hline 1.2 & 0.1966 & 0.1078 & 0.0604 & 0.0059 \\
\hline 1.4 & 0.1554 & 0.0809 & 0.0421 & 0.0028 \\
\hline 1.6 & 0.1172 & 0.0579 & 0.0279 & 0.0013 \\
\hline 1.8 & 0.0856 & 0.0399 & 0.0177 & 0.0005 \\
\hline 2.0 & 0.0609 & 0.0267 & 0.0109 & 0.0002 \\
\hline 3.0 & 0.0085 & 0.0026 & 0.0007 & 0.0000 \\
\hline 4.0 & 0.0009 & 0.0002 & 0.0003 & 0.0000 \\
\hline 5.0 & 0.0001 & 0.0000 & 0.0000 & 0.0000 \\
\hline
\end{tabular}


Tables 7.2 and 7.4 show that the damping coefficients $\lambda_{11}$ and $\lambda_{33}$ start from zero and after a certain value of $K a$, they decrease uniformly to reach zero again when $K a=5.0$. Also, we notice that the damping coefficients are smaller compared to the added-mass for all the submergence values.

Analytic expressions for the total forces have been determined in Section 6. The effects of diffraction and radiation have been accounted for. The research in this area is continuing. Our next step will be to display the results in graphical form considering the effects of diffraction, the effects of radiation, and the combined effects of diffraction and radiation on a submerged sphere in regular waves of finite water depth. These results , if possible, will be compared with the available experimental or field data. We are very enthusiastic in our goal to collect field data to confirm this mathematical theory.

ACKNOWLEDgement. The author is grateful to Natural Sciences and Engineering Research Council (NSERC) of Canada for the financial support leading to this paper.

\section{REFERENCES}

[1] S. N. Bora, The interaction of water waves with submerged spheres and circular cylinders, Ph.D. thesis, Technical University of Nova Scotia, Halifax, Canada, 1997.

[2] S. N. Bora, M. Rahman, and M. G. Satish, Recent mathematical developments of potential theory applied to diffraction of water waves, Potential Flow of Fluids, Adv. Fluid Mech., vol. 6, Comput. Mech., Southampton, 1995, pp. 199-243. MR 96h:76005.

[3] E. P. Gray, Scattering of a surface wave by a submerged sphere, J. Engrg. Math. 12 (1978), no. 1, 15-41. MR 57\#2104. Zbl 0399.76020.

[4] T. H. Havelock, The wave resistance of a spheroid, Proc. Roy. Soc. London Ser. A 131 (1931), 275-285. Zbl 0001.42301.

[5] A. Hulme, The wave forces acting on a floating hemisphere undergoing forced periodic oscillations, J. Fluid Mech. 121 (1982), 443-463. MR 83k:76013. Zbl 0492.76022.

[6] N. W. McLachlan, Bessel Functions for Engineers, Oxford University Press, UK, 1941.

[7] T. Sarpkaya and M. Isaacson, Mechanics of Wave Forces on Offshore Structures, Van Nostrand Reinhold Company, New York, 1981.

[8] R. C. Thorne, Multipole expansions in the theory of surface waves, Math. Proc. Cambridge Philos. Soc. 49 (1953), 707-716. MR 15,361g. Zbl 0052.43106.

[9] S. Wang, Motions of a spherical submarine in waves, Ocean Eng. 13 (1986), 249-271.

[10] G. X. Wu and R. E. Taylor, The exciting force on a submerged spheroid in regular waves, J. Fluid Mech. 182 (1987), 411-426. Zbl 0633.76019.

[11]___ On the radiation and diffraction of surface waves by submerged spheroids, J. Ship Research 33 (1989), 84-92.

[12] G. X. Wu, J. A. Witz, Q. Ma, and D. T. Brown, Analysis of wave induced forces acting on a submerged sphere in finite water depth, Applied Ocean Research 16 (1994), 353361.

MATIUR RAHMAN: DePARTMENT OF ENGINEERING MATHEMATICS, DALHOUSIE UniVERSITY, P.O. Box 1000, HalifaX, Nova Scotia, CANADA B3J 2X4

E-mail address: matiur.rahman@da1.ca 


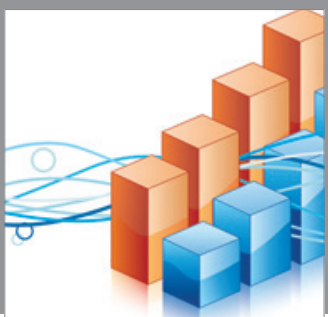

Advances in

Operations Research

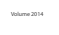

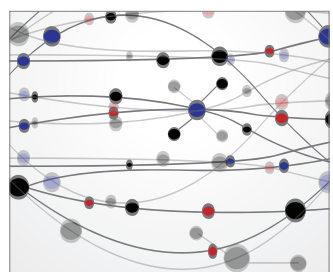

\section{The Scientific} World Journal
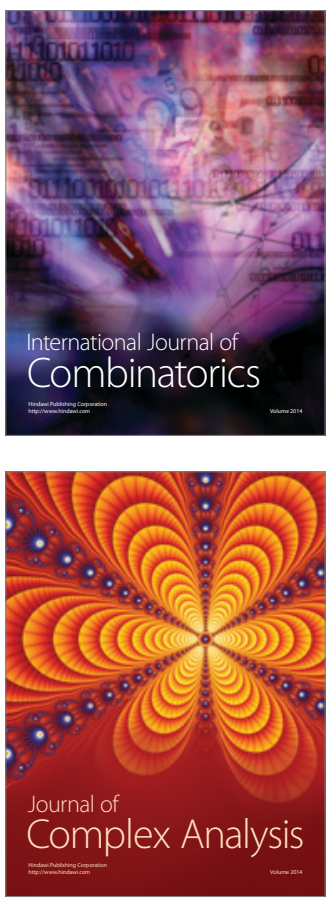

International Journal of

Mathematics and

Mathematical

Sciences
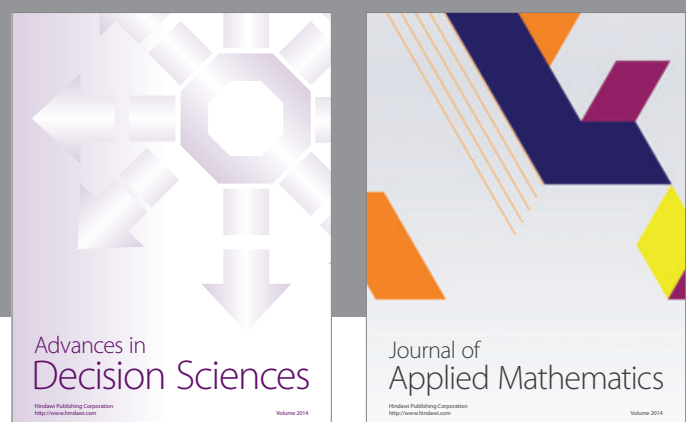

Journal of

Applied Mathematics
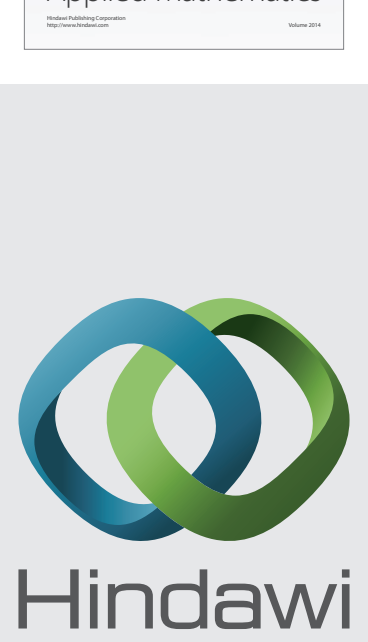

Submit your manuscripts at http://www.hindawi.com
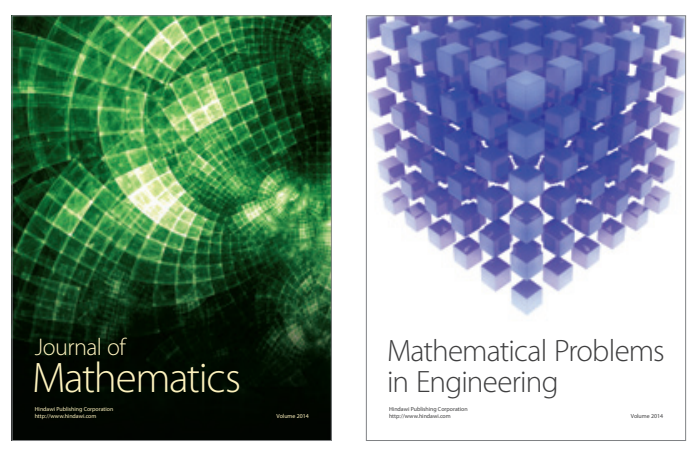

Mathematical Problems in Engineering
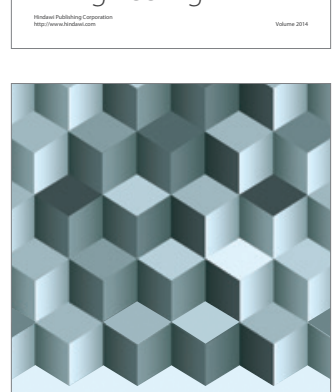

Journal of

Function Spaces
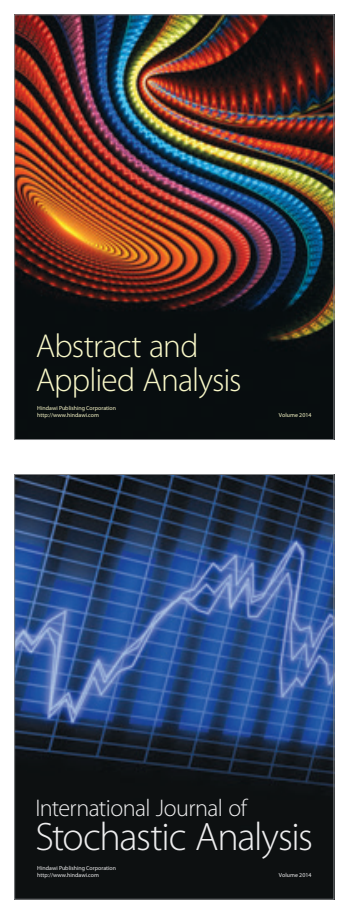

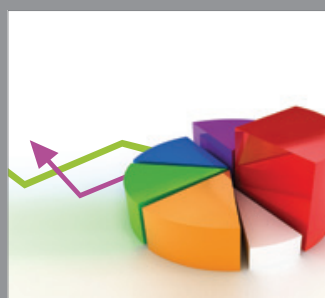

ournal of

Probability and Statistics

Promensencen
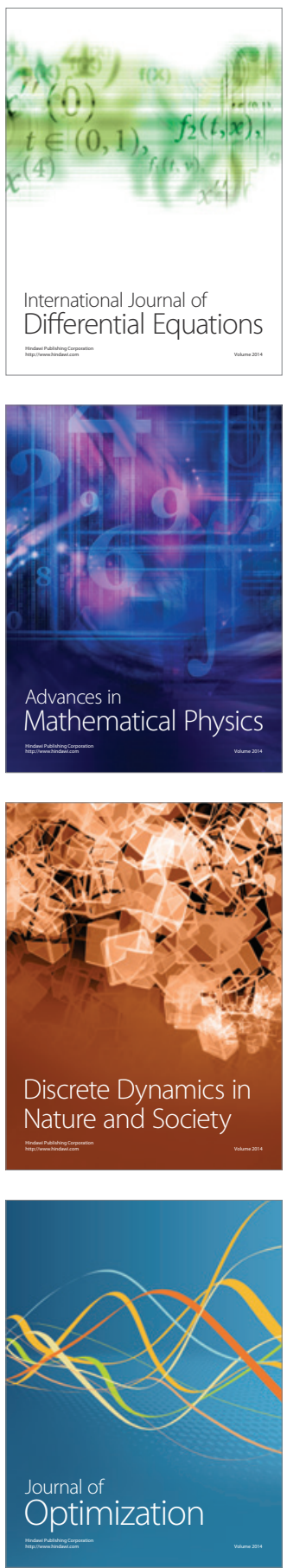